\title{
Description de deux usages non subordonnants de la forme alors que
}

\author{
Lafontaine, Fanny \\ Aix-Marseille Université, Laboratoire Parole et Langage (UMR 7309) \\ fanny.adamlafontaine@gmail.com
}

\section{Introduction}

Dans les grammaires traditionnelles, les séquences introduites par la forme alors que se conçoivent uniquement sous le statut de la « subordination ${ }^{1} »$. En effet, cette forme est traitée comme une conjonction de subordination à valeur temporelle dominante, assortie ou non d'une nuance d'opposition, telle qu'illustrée en (1) :

(1) Je l'ai vue deux ou trois fois alors que j'étais à Toulouse (Grevisse, M. \& Goosse, A., 2008 : 1482)

Or, il apparaît que, dans les corpus de français écrit et oral ${ }^{2}$, on rencontre certains emplois qui ne peuvent être ramenés à des faits de dépendance grammaticale dès qu'on examine précisément leurs propriétés syntaxiques; les séquences introduites par la conjonction alors que peuvent connaître d'autres fonctionnements syntaxiques ${ }^{3}$, associés à divers effets de sens tels que le contraste (2) et (3) ou la concession (4) et (5) :

(2) à quinze ans un chien il est déjà très vieux + alors que nous + on dit qu'on est très vieux + on commence à être très vieux qu'à partir de quatre-vingts ans (oral, Corpaix)

(3) D'un côté, les chasseurs paient pour exercer dans la nature leur passion qui est de réussir de beaux "cartons" alors que les autres chasseurs (champignons, photos, etc.) ne paient rien (écrit, Cerf)

(4) Il a fini par l'écœurer - il le payait au S.M.I.G. alors qu'il faisait - des horaires que je fais moi patron c'est-à-dire trente cinq heures tous les deux jours (oral, Corpaix)

(5) Peu d'animaux sont aussi calomniés que la belette. Alors qu'on devrait la tolérer et même la protéger, on la persécute partout et en tout temps (écrit, Cerf)

Dans une première partie, nous présenterons le modèle de description qui a guidé cette étude en rappelant les propriétés qui permettent de distinguer les emplois subordonnants classiques dans lesquels la conjonction instaure une relation de dépendance syntaxique (1) et les emplois non subordonnants pour lesquels la relation instaurée est discursive et non plus syntaxique (2-5). Puis, nous montrerons qu'à l'intérieur de ces emplois non subordonnants, il est possible de discriminer deux types d'organisations remarquables sur la base de leurs propriétés syntaxiques.

\section{Présentation du cadre d'analyse}

Le cadre de description qui fondera ce travail délaissera la notion de «subordination » pour étudier les relations de dépendance syntaxique à partir de la notion plus générale de «rection syntaxique». L'approche pronominale, définie dans l'ouvrage «Pronom et syntaxe», (C. Blanche-Benveniste et al., 1984) décrit les éléments organisés par le verbe en ayant recours aux paradigmes de pronoms ou proformes. D'autres critères liés également à l'insertion paradigmatique permettent de vérifier le lien de dépendance syntaxique entre un verbe recteur et un élément de la construction: le clivage, le contraste de modalités, la reprise par et cela ou encore l'insertion d'un 
adverbe paradigmatisant. En conséquence, la relation de dépendance entre un verbe et une «subordonnée circonstancielle » sera définie sur la base de ces critères formels et non sur le simple statut catégoriel de l'élément introducteur. A partir de cette première série de tests, nous allons illustrer la distinction fondamentale qu'il existe entre les emplois régis et non régis.

\subsection{Alors que introducteur d'une séquence régie.}

Nous considérons qu'en (6) la séquence à valeur temporelle introduite par alors que est régie par le verbe arriver et que l'ensemble forme une seule construction verbale puisque l'équivalence à une proforme est dans notre modèle de description un indice fort de rection (6a) :

(6) [Ils arrivèrent sous les murailles de la puissante Ilion alors que la nuit tombait. $]_{\text {Constr. (écrit, }}$ Cerf)

(6a) Ils arrivèrent sous les murailles de la puissante Ilion à ce moment-là

Néanmoins, ces séquences ont la particularité de résister à certains tests d'insertion paradigmatique et présentent ainsi, hormis quelques attestations issues principalement du web, des limitations que ne connaissent habituellement pas les autres types de constituants régis à valeur temporelle, tels que ceux en quand ou lorsque.

Comme première limite, cette séquence ne peut répondre à une question en quand (6b) :

(6b) Quand est-ce qu'ils arrivèrent sous les murailles de la puissante Ilion? * Alors que la nuit tombait

De plus, on ne rencontre pas dans les corpus de construction en alors que qui soit clivée (6c) :

(6c) * c'est alors que la nuit tombait qu'ils arrivèrent sous les murailles de la puissante Ilion

Cependant, les séquences en alors que peuvent être prises dans une tournure clivée si elles apparaissent en seconde position, par exemple à la suite d'un syntagme introduit par «pendant» :

(7) C'est pendant la troisième nuit, alors qu'il s'était installé sur le petit sommet d'un énorme rocher à fleur de versant, dans une trouée parmi les arbres noirs et tristes, qu'il sentit les premiers effets de la solitude sur sa perception de la forêt. (écrit, Cerf)

En outre, il semble que le contraste de modalités en «non pas alors que $\mathrm{X}$ mais alors que $\mathrm{Y}$ » soit quasiment irrecevable (6d) :

(6d) ? Ils arrivèrent sous les murailles de la puissante Ilion non pas alors que la nuit tombait mais alors que le jour se levait

On relève toutefois quelques cas où la séquence en alors que admet l'opposition avec d'autres formes de circonstances temporelles :

(8) Cependant, il est décédé à Vienne, non pas alors que la paix était rétablie dans l'Empire, mais à la veille d'une nouvelle campagne. (web)

(9) Le sevrage à 25 - 30 jours se déroule non pas quand la truie produit déjà moins de lait mais alors que les porcelets continuent de téter et que la production continue d'être très élevée (8-10 kg par jour). (web)

(10) Selon les premiers éléments de l'enquête, le prêtre aurait été heurté par la cloche soit alors qu'il la sonnait, soit après un malaise. (écrit, Cerf)

L'insertion d'un adverbe paradigmatisant (6e) n'est guère attestée avec ce type de forme :

(6e) ? Ils arrivèrent sous les murailles de la puissante Ilion surtout alors que la nuit tombait. 
On la rencontre assez naturellement dans l'exemple (11) où on a une énumération de circonstances temporelles :

(11) Londres arrive au Japon à la fin de 1921 et y restera quelques mois. Alors que l'hiver est déjà entamé, alors que Monsieur Paul Claudel est ambassadeur à Tokyo, mais surtout alors que la conférence de Washington bat son plein et que les Etats-Unis et l'Angleterre s'apprêtent à infliger une nouvelle humiliation aux nippons. (web)

Bien que la reprise par et cela (6f) ne nous semble pas agrammaticale, on n'en trouve aucune occurrence dans les corpus :

(6f) ? Ils arrivèrent sous les murailles de la puissante Ilion et cela alors que la nuit tombait

Seul, l'internet nous en offre quelques cas :

(12) Il ne s'agit pas seulement de viser une rentabilité économiqueà tout prix mais de répondre au défi que constitue l'alimentation de tous, et cela alors que la population mondiale ne cesse de croitre. (web)

A la différence des séquences en alors que, les séquences à valeur temporelle introduites par quand ne connaissent pas ces restrictions car, outre leur proportionnalité à la proforme «à ce moment-là », elles peuvent être interrogées par «quand»(13), clivées (14), contrastées (15), modifiées par un adverbe paradigmatisant (16) et enfin être précédées par « et cela » (17) :

(13) Alors, je lui dis : "... Les gradins... vous les démontez quand ?" " Quand la mer sera remontée. " " Vous la remontez quand, la mer ?" Il me dit : " Quand vous serez parti ! " (écrit, Cerf)

(14) C'est ça, c'est toujours ces garçons qui vous font du chantage affectif en fin de compte, et c'est quand on s'en va qu'ils s'rendent compte de votre valeur, voilà ! (écrit, Cerf)

(15) cela signifie que votre chien mangera après vous, et mangera quand vous l'avez décidé et non pas quand lui en a envie comme dans un libre service. (écrit, Cerf)

(16) c'est toujours embêtant surtout quand on travaille » (oral, Corpaix)

(17) Pas un nuage à l'horizon, et cela quand il y a tant à faire dans la science de l'artillerie ! (écrit, Cerf)

Enfin, il est à noter que les séquences en alors que régies ne présentent pas de contrainte de position, tout comme pour quand ou lorsque, et peuvent figurer en tête d'énoncé :

(18) Alors que leurs employeurs n'ont toujours pas versé le premier centime de cotisations, payables en avril, les gardes d'enfants trinquent déjà. (écrit, Cerf)

On peut conclure que la structure en alors que est intégrée à la construction verbale par les liens de la rection, et ce malgré les résistances à certains tests d'insertion paradigmatique, lesquelles semblent inhérentes à cette forme ${ }^{4}$. Outre cet emploi de marqueur d'une rection temporelle, les autres usages de alors que, dont nous allons présenter à la suite deux types, mettent en jeu des structures discursives.

\subsection{Alors que introducteur de séquences non régies}

Dans les exemples (2-5) ainsi qu'en (19) et (20) ci-dessous, la construction introduite par alors que ne peut en rien être ramenée à des faits de dépendance grammaticale par rapport au verbe de la construction précédente.

(19) La Sotrasi a refusé de renouveler les filtres de nos masques pendant une semaine alors qu'il faut les changer normalement chaque jour. (écrit, Cerf) 
(20) sur l'Acropora c'est un petit polype alors que sur le Catalaphyllia - c'est des gros polypes (oral, CRFP)

Contrairement au fonctionnement régi analysé précédemment, il n'est pas possible dans ces cas de réduire la séquence en alors que à une proforme.

De plus, le clivage produit des énoncés agrammaticaux :

(19a) * c'est alors qu'il faut les changer normalement chaque jour que la Sotrasi a refusé de renouveler les filtres de nos masques pendant une semaine

$(20 a) *$ c'est alors que sur le Catalaphyllia c'est des gros polypes que sur l'Acropora c'est un petit polype

En outre, on ne rencontre aucune séquence dans les corpus qui soit organisée en couples contrastifs :

(19b) * La Sotrasi a refusé de renouveler les filtres de nos masques pendant une semaine non pas alors qu'il faut les changer normalement chaque jour mais alors que...

(20b) * sur l'Acropora c'est un petit polype non pas alors que sur le Catalaphyllia c'est des gros polypes mais alors que...

De la même manière que pour les emplois régis, on ne peut les faire précéder d'un adverbe paradigmatisant :

$(19 \mathrm{c}) *$ La Sotrasi a refusé de renouveler les filtres de nos masques pendant une semaine surtout alors qu'il faut les changer normalement chaque jour.

$(20 \mathrm{c}) *$ sur l'Acropora c'est un petit polype surtout alors que sur le Catalaphyllia c'est des gros polypes.

Seul le test de la reprise par « et cela » nous fournit des résultats différents entre les deux séquences ; dans celle donnée en (19), qui illustre l'emploi « concessif» de la conjonction, cette reprise est possible :

(19d) La Sotrasi a refusé de renouveler les filtres de nos masques pendant une semaine et cela alors qu'il faut les changer normalement chaque jour.

On en relève quelques occurrences dans les corpus :

(21) Nicole Kidman serait en effet tombée enceinte de Tom Cruise à peu près au moment de leur séparation, soit à l'aube de leurs dix années de mariage et cela, alors que l'acteur n'avait jamais pu jusque-là avoir d'enfant. (écrit, Cerf)

En revanche, la séquence (20), dans laquelle la conjonction est dotée d'une valeur «contrastive », ne supporte pas le détachement par « et cela »:

$(20 \mathrm{~d}) *$ sur l'Acropora c'est un petit polype et cela alors que sur le Catalaphyllia c'est des gros polypes

Si pour (19) le test de la reprise par « et cela » est insuffisant pour attester d'un quelconque lien de nature rectionnelle, il permet néanmoins de montrer en premier lieu que ces deux séquences non régies -(19) et (20)- sont susceptibles de relever de deux fonctionnements distincts.

A présent qu'a été établie une première opposition entre emplois régis et non régis, nous allons affiner la description de ces derniers en montrant qu'ils relèvent bien de deux fonctionnements syntacticosémantiques discriminants.

\section{Description de deux classes de non régis}

Nous proposons d'indiquer les propriétés syntaxiques qui permettent de singulariser les deux usages de alors que non régis illustrés ci-dessus, que nous désignerons comme le «type concessif » et le «type contrastif », et ce après avoir évoqué leur dimension sémantique. 


\subsection{Le type de relation sémantique}

Dans l'exemple donné en (20), alors que signale une relation de contraste où « deux entités distinctes se voient assigner des propriétés qui s'excluent mutuellement dans le contexte» (W. Spooren, 1989 : 71). Les deux référents "l'Acropora » et « le Catalaphyllia » sont opposés quant à la taille de leurs polypes (petit vs. gros). K. Sandfeld (1965) relève dans cet usage que «'tandis que' [et] 'alors que'..., se rapprochent du sens de 'par contre'.» (p.305) D'autres marqueurs comme mais ou en revanche entrent dans le paradigme sémantique de alors que :

(20e) sur l'Acropora c'est un petit polype par contre/mais/en revanche sur le Catalaphyllia c'est des gros polypes

Comme le note C. Roze (2013: 72), dans les configurations contrastives, «soit les propriétés incompatibles ${ }^{5}$ font directement partie du contenu des unités reliées -comme en (20) ou (22)- soit une des propriétés est inférée à partir du contenu d'une des unités reliées- comme en (23). »

(22) les Streptocoques pousseront alors que la plupart des autres bactéries ne pousseront pas (oral, Corpaix)

(23) Le jour de la mort de Georges Brassens, j'ai pleuré, alors que le jour de la mort de Tino Rossi... J'ai repris deux fois des moules ! (écrit, Cerf)

Dans cet usage, la relation contrastive s'incarne le plus souvent sous la forme de parallélismes syntaxiques. A ce propos, C. Blanche-Benveniste et al. (1990 : 181) relèvent que «lorsque, dans deux énoncés successifs, on retrouve le même schéma syntaxique, c'est souvent avec un effet de contraste ; les lexèmes placés dans les mêmes positions syntaxiques contrastent entre eux : moi je jouais dans un orchestre elle elle chantait euh elle dans les cours »

Enfin, dans les cas contrastifs, on peut renverser l'ordre des deux constructions verbales sans que cela ne modifie le sens de l'énoncé car elles sont sémantiquement symétriques :

(20f) sur le Catalaphyllia c'est des gros polypes alors que sur l'Acropora c'est un petit polype

Concernant l'exemple (19), on l'interprète comme concessif ${ }^{6}$ car les deux structures reliées par alors que relèvent cette fois-ci d'une contradiction et non plus d'une comparaison : par mesure de sécurité, il est obligatoire de changer quotidiennement les filtres or la Sotrasi s'y refuse. Dans ce type de contexte, des formes comme bien que, malgré le fait que ou en dépit du fait que sont des équivalents sémantiques de alors que :

(19e) La Sotrasi a refusé de renouveler les filtres de nos masques pendant une semaine bien $\mathrm{qu}^{\prime} / \mathrm{malgré}$ le fait qu'/ en dépit du fait qu'il faille les changer normalement chaque jour.

Par opposition au type contrastif, la relation concessive est asymétrique, c'est-à-dire que le renversement de l'ordre des constructions verbales conduit à un énoncé sémantiquement douteux :

(19f) * il faut changer les filtres de nos masques normalement chaque jour alors que la Sotrasi a refusé de les renouveler pendant une semaine

\subsection{Le statut paradigmatique et syntagmatique}

Au plan grammatical, nous évoquerons d'abord le mode de réalisation des énoncés afin de distinguer les deux types de non régis : alors que contrastif exploite la relation paradigmatique -de la même manière que certains usages du connecteur mais- et alors que concessif la relation syntagmatique.

L'usage de alors que contrastif s'apparente aux « listes paradigmatiques» en étant compatible avec les structures à projection, qui correspondent à une «organisation lexicale bis de la relation syntaxique 
donnée préalablement » et qui s'instaurent par la présence d'«au moins deux éléments appartenant à deux paradigmes différents de la construction verbale» (C. Blanche-Benveniste et al., 1990 : 152).

Ainsi, on pourrait simplifier la syntaxe interne de la construction introduite par alors que à deux éléments, l'un réalisant une place syntaxique et l'autre une modalité de relation qui s'interpréterait relativement à ce qui précède ; à partir de (20), en projetant la seconde construction sur la première, on aurait cette équivalence avec l'élément locatif là et la modalité de relation non :

sur l'Acropora

c'est un petit polype

alors que

là

non

On rencontre dans les corpus ce type de phénomène :

(24) E1 maintenant nous on entend p-

E1 des gens qui parlent que provençal ça existe plus

E2 non non

E1 quoi alors qu'avant oui ouais (oral, CRFP)

On relève également quelques cas particuliers de projection pour lesquels on a affaire à une élision de la construction verbale de la séquence projetée mais où, par le jeu des contrastes lexicaux en référence aux positions syntaxiques ouvertes dans la construction verbale précédente, on peut interpréter facilement la partie élidée :

(25) A votre avis pourquoi l'Etat n'impose pas plus les riches ? Gagné ! = parce qu'ils s'en iraient voir si l'herbe est plus verte ailleurs alors que nous exploités ici ou ailleurs... et les entreprises de luxe alors pour qui elles tourneraient ! (écrit, Cerf)

(26) "Mais toi, tu détestais ta mère, alors que moi je l'aimais, c'est donc moi qui souffre le plus."C'est ce que tu crois, s'empressa d'ajouter le premier, la tienne n'a pas eu à subir d'opération, elle. Elle est morte sans souffrir, alors que la mienne... "'Oui mais la tienne n'a rien senti, n'a pas su ce qui lui arrivait, alors que la mienne... " (écrit, Cerf)

Un autre argument permet d'attester du caractère paradigmatisable d'une suite de constructions: la possibilité qu'elles soient enchâssées par un verbe et qu'elles réalisent une même position syntaxique pronominalisable par une forme unique. Dans l'exemple (27), la reprise de la conjonction que après la forme mais est un indice fort de dépendance grammaticale et de mise en liste d'un élément de valence :

(27) Sache que l'union fait la force mais que la misère la divise (chanson)

Cet énoncé peut être réorganisé de cette manière :

Sache

que l'union fait la force

mais

que la misère la divise

ceci

En (28) et (29), les deux constructions verbales articulées par alors que et enchâssées par les verbes «montrer » et « estimer» réalisent sous forme de listes une position d'objet direct; comme mais en (27), alors que a un fonctionnement de joncteur de listes:

(28) cela m'a montré que des volontaires résolvent eux-mêmes leurs problèmes alors que les appelés attendent qu'on les règle à leur place. (écrit, Cerf)

(29) on peut estimer que le RPR et le FN sont à égalité. Avec, chacun, 50000 militants. Et que le premier chute, alors que le second progresse. " (écrit, Cerf) 
(28a) cela m'a montré

(29a) On peut estimer

alors que

que des volontaires résolvent eux-mêmes leurs problèmes

alors que (que) les appelés attendent qu'on les règle à leur place

ceci

que le RPR et le FN sont à égalité

que le premier chute

(que) le second progresse

ceci

A la différence de mais, le que complémenteur n'est pas présent en surface dans cette configuration mais si les éléments de la liste sont énoncés isolément, que sera bien réalisé :

(28b) cela m'a montré que des volontaires résolvent eux-mêmes leurs problèmes // cela m'a montré que les appelés attendent qu'on les règle à leur place

Ce type de réduction n'est pas $a d-h o c$, mais se trouve indépendamment justifié. Les grammaires relèvent en effet ce phénomène sous le terme d' « haplologie » en citant entre autres l'exemple de la forme "plutôt que » (Grevisse, M. \& Goosse, A., $2008: 1563)$ :

(30) Je préfère que tu attendes un an plutôt que tu en attendes trois.

$$
\begin{aligned}
& \text { Je préfère que tu attendes un an } \\
& \text { plutôt que (que) tu en attendes trois }
\end{aligned}
$$

ceci

Nous pouvons donc maintenir que alors que dans ces exemples est impliqué dans une relation paradigmatique.

Dans les séquences à valeur concessive, il est impossible de projeter la seconde construction sur la première car alors que n'introduit pas de nouveau constituant pouvant être projeté sur la place correspondant à « la Sotrasi » :

$$
(19 \mathrm{~g}) *
$$

La Sotrasi a refusé de renouveler les filtres de nos masques pendant une semaine

alors que ?

oui

L’élision de la séquence introduite par alors que produit un énoncé tronqué :

$(19 \mathrm{~h}) *$ La Sotrasi a refusé de renouveler les filtres de nos masques pendant une semaine alors que...

Aussi, l'énoncé concessif relié par la forme alors que peut dans certains contextes former un seul constituant qui occupe une place essentielle par rapport au verbe recteur :

(31) raconter des contes de fées à des adultes alors que la situation à Paris se dégrade ostensiblement n'est peut-être pas du meilleur à propos (écrit, Cerf)

(32) Je trouve tout à fait normal qu'une société fasse des bénéfices en améliorant ses méthodes de production, en élargissant sa gamme de produits ou son réseau de distribution, mais clamer de 
bonnes performances alors que celles-ci sont avant tout dues à des facteurs économiques extérieurs est dérangeant. (écrit, Cerf)

En (31) et (32), on peut en premier lieu mettre en évidence ce regroupement par les tests de la pronominalisation (a) et du pseudo-clivage (b) :

(31a) ceci n'est peut-être pas du meilleur à propos

(32a) ceci est dérangeant

(31b) ce qui n'est peut-être pas du meilleur à propos c'est de raconter des contes de fées à des adultes alors que la situation à Paris se dégrade ostensiblement.

(32b) ce qui est dérangeant c'est de clamer de bonnes performances alors que celles-ci sont avant tout dues à des facteurs économiques extérieurs

On en déduit que, contrairement aux types contrastifs décrits ci-dessus où la position d'objet était instanciée sous forme de liste de constructions verbales, en (31) et (32), la fonction sujet n'est réalisée qu'une seule fois par une association de constructions verbales ${ }^{7}$. En second lieu, le fonctionnement de alors que ne peut en rien être assimilé à celui d'un joncteur de liste articulant deux constructions verbales réalisables indépendamment, car il est impossible ici pour la séquence «la situation à Paris se dégrade ostensiblement » d'occuper seule cette fonction sujet (31c), signe que les deux constructions verbales reliées par alors que entretiennent un rapport syntagmatique et non paradigmatique (31d) :

(31c) * la situation à Paris se dégrade ostensiblement n'est pas du meilleur à propos.

raconter des contes de fées à des adultes

n'est pas du meilleur à propos

alors que la situation à Paris se dégrade ostensiblement

Ceci

\subsection{Le critère de position}

Dans les cas contrastifs, alors que qui introduit une nouvelle unité de discours faisant suite à un énoncé précédent présente une contrainte discursive de positionnement à droite :

(20h) * alors que sur le Catalaphyllia c'est des gros polypes sur l'Acropora c'est un petit polype

Cette contrainte pourrait découler d'un fonctionnement comme connecteur discursif de alors que qui serait alors comparable à celui de l'adverbial par contre. Mais, contrairement à ce dernier, alors que apparait nécessairement à la jointure des deux constructions verbales:

(33) Miller prend en effet cela très au sérieux. En France, par contre la réception fut différente. (écrit, Cerf)

(33a) * Miller prend en effet cela très au sérieux. En France, alors que la réception fut différente

Alors que a donc les contraintes de position d'une conjonction de coordination, tout comme et et mais contrastifs.

Dans les cas concessifs, la forme alors que ne présente pas de contrainte de position et peut aussi bien être postposée (19) que antéposée (34) :

(34) Alors que ces enseignants ont exercé, pendant des années, dans des conditions difficiles leur métier, 11000 d'entre eux se retrouvent aujourd'hui au chômage (écrit, Cerf) 


\subsection{La portée des modalités}

La valeur illocutoire de la séquence en alors que contrastive peut être différente de celle de la construction précédente même si de tels exemples de variation de modalité sont assez rares dans les données. En (35) et (36), on relève la présence d'une modalité interrogative ${ }^{8}$ qui fait suite à une première assertion :

(35) Les humains peuvent s'hydrater, se protéger, se ressourcer en changeant de place et en se rendant vers des cieux plus cléments. Alors que lui, que peut-il faire si ce n'est du bout de ces racines, tenter de récupérer le peu d'humidité qui stagne encore sous terre ? (web)

(36) On se rend compte que deux enfants sur trois connaissent ces jeux, un sur quatre l'a déjà vu pratiqué alors que les parents les parents est-ce que deux parents sur trois connaissent bien ces jeux est-ce que un sur quatre l'ont vu pratiqué je pense pas (oral, corpus personnel)

L'exemple à valeur contrastive suivant débutant par une modalité interrogative ne peut affecter la séquence en alors que :

(37) Qui se souvient encore de Lendl, aujourd'hui, malgré son palmarès peut-être supérieur à celui de son adversaire, alors que tout le monde admire encore McEnroe. (écrit, Cerf)

En effet, grâce au test de la permutabilité des constructions verbales, on se rend compte qu'on a bien affaire à deux modalités différentes, une interrogative et une déclarative, et non à une seule qui serait couvrante :

(37a) tout le monde admire encore McEnroe alors que qui se souvient encore de Lendl, aujourd'hui, malgré son palmarès peut-être supérieur à celui de son adversaire

En revanche, les structures concessives ont la possibilité de se trouver dans la portée d'une interrogation ; J.-M. Debaisieux (2004: 126) relève ce fait « lorsque les [deux propositions concessives articulées par bien que] constituent 'un seul acte de parole'»:

(38) Est-ce qu'elle est insuffisante bien qu'elle soit confirmée par le séjour en Amérique ?

Les séquences en alors que présentent cette même possibilité d'être couvertes par une interrogative ${ }^{9}$ (39) et (40), comme le montre la possibilité pour les deux constructions reliées par cette forme d'être pronominalisées par le clitique «le» $(39 \mathrm{a})$ :

(39) Comment pourrait-il m'inviter moi alors que j'ai la figure pleine de ces horribles boutons ? (écrit, Cerf)

(40) Comment ont-ils pu se ranger du côté des militaires alors qu'il n'y avait plus de risque politique depuis 20 ans ? (écrit, Cerf)

(39a) Comment le pourrait-il ? [m'inviter moi alors que j'ai la figure pleine de ces horribles boutons] :

Si les deux constructions se trouvent désolidarisées de sorte que celle introduite par alors que est détachée en tête de structure et qu'elle n'est donc plus prise en compte par le clitique «le", cela a pour conséquence de modifier le sens de l'énoncé. En effet, dans ces cas d'antéposition, la séquence en alors que fait mention d'un fait fonctionnant comme cadre justifiant l'acte de réfutation contenu dans l'interrogation oratoire qui suit.

(39b) alors que j'ai la figure pleine de ces horribles boutons, comment le pourrait-il ? [m'inviter moi]

K. Sandfeld (1965) classe la forme alors que dans ces tours parmi les conjonctions causales, considérant une équivalence avec des formes comme vu que ou étant donné que : 
(39c) étant donné que j'ai la figure pleine de ces horribles boutons, comment pourrait-il m'inviter ?

Ainsi, l'affectation ou non par la portée de l'interrogation de la séquence en alors que a donc une incidence sur l'interprétation de l'énoncé.

\subsection{Le critère de contiguïté}

Les structures contrastives se caractérisent en outre par le fait que sur le plan prosodique chaque construction peut être réalisée indépendamment. A l'écrit, un «point» permet de signifier cette absence de ligature mélodique :

(41) les journaux ont principalement retenu deux choses de cette première journée : pour la presse d'outre-Manche, le fait du jour est sans conteste la nouvelle querelle franco-britannique sur la force de réaction rapide européenne. Alors que les journaux espagnols et italiens reviennent plutôt sur la signature de la Charte des droits fondamentaux. (écrit, Cerf)

Par ailleurs, on constate dans les données orales une grande distance entre la première construction verbale et la seconde introduite par alors que. Dans l'exemple qui suit, cette absence de contiguité est à mettre en lien avec la présence de l'élément cadratif «en fin d'année » au sujet duquel plusieurs constructions verbales sont développées :

(42) et en fin d'année on fait une autre une autre soirée c'est plutôt le thème là euh sur l'extérieur c'est plus danse(s) folk(s) là parce qu'on essaie de faire dans ça dans un champ ou dans un terrain - et là c'est les brochettes c'est les et puis on danse les danses folks et on entraîne tout le monde dans des danses - un petit peu comme autrefois là donc encore - ça c'est des sorties elles marchent très bien nos - nos soirées folks parce que - c- ça a quand même plus à voir avec le culturel du coin alors que les - en février bon bé c'est les gens qui vont de de - de soirées de salons de thé en salons de thé /toute la, XXX/ soirée comme ça et qui qui se qui se baladent quoi ils viennent danser uniquement hein - (oral, CRFP)

Dans tous les cas de alors que concessif, aucune unité syntaxique ne vient s'intercaler entre les deux séquences; il y a donc une stricte contiguité. On peut toutefois rencontrer à l'écrit une ponctuation forte entre les deux constructions :

(43) De son côté, le gouvernement s'apprête à revoir la réglementation dans un décret. " Insuffisant ", juge la CFDT au vu du projet. Celui-ci ne prévoit qu'un usage contrôlé des solvants et l'interdiction aux femmes enceintes d'être exposées aux éthers de glycol. Alors que les scientifiques estiment que les risques de malformation existent dès la conception. (écrit, Cerf)

Mais de telles ruptures prosodiques sont attestées avec des constructions régies par le verbe d'une unité précédente : il s'agit du phénomène d'épexégèse.

\section{Conclusion}

Comme cela a déjà été montré pour d'autres conjonctions, la forme alors que ne peut être traitée d'une manière unifiée. En effet, au-delà du repérage de ses divers effets de sens, nous avons abordé la question de ses différents statuts syntaxiques. Les tests d'insertion paradigmatique s'avèrent fructueux en ce qu'ils permettent d'établir une première distinction entre ce qui relève d'une part de la dépendance grammaticale et d'autre part de l'enchaînement discursif. A partir de là, nous avons vu que, outre l'emploi rectionnel qui correspond au complément circonstanciel à valeur temporelle de l'analyse traditionnelle, les autres cas de alors que-contrastifs et concessifs- ne pouvaient être ramenés à des faits de dépendance rectionnelle. Un certain nombre de propriétés nous a permis de rendre compte d'une dichotomie très nette entre les deux types de alors que non régis ; si dans les cas à valeur contrastive, le comportement de alors 
que est assimilable à celui d'une conjonction de coordination eu égard à certaines de ses propriétés (sa contrainte de positionnement à droite et son statut paradigmatique), en revanche, dans les cas à valeur concessive, on observe une forme de rattachement plus forte entre les deux constructions verbales reliées par alors que.

\section{Tableau récapitulatif des propriétés des deux non régis}

\begin{tabular}{|c|c|c|c|c|c|}
\hline Type Propriétés & $\begin{array}{c}\text { Reprise par } \\
\text { « et cela » }\end{array}$ & Paradigmatisable & $\begin{array}{c}\text { Contrainte } \\
\text { de position }\end{array}$ & $\begin{array}{c}\text { Portée des } \\
\text { modalités }\end{array}$ & $\begin{array}{c}\text { Absence } \\
\text { de } \\
\text { contigüité }\end{array}$ \\
\hline Contrastif & - & + & + & - & + \\
\hline Concessif & + & - & - & + & - \\
\hline
\end{tabular}

\section{Références bibliographiques}

Benzitoun, C. (2008). Qui est quand ? Essai d'analyse catégorielle. Langue Française, 158, 129-143.

Blanche-Benveniste, C., Deulofeu J., Stéfanini J. et Van Den Eynde K. (1984). Pronom et syntaxe. L'approche pronominale et son application au français, Paris : SELAF.

Blanche-Benveniste, C., Bilger M., Rouget C., Van Den Eynde K. et Mertens P. (1990). Le Français parlé : études grammaticales. Paris : Ed. du CNRS.

Blumenthal, P. (1980). La syntaxe du message. Application au français moderne. Tübingen : Max Niemeyer Verlag.

Charolles, M. (2003). De la topicalité des adverbiaux détachés en tête de phrase. Travaux de linguistique, 47, 11-51.

Debaisieux, J.-M. (2004). Quel statut syntaxique pour les propositions «subordonnées circonstancielles »? La distinction entre dépendance grammaticale et dépendance macro-syntaxique comme moyen de résoudre les paradoxes de la subordination. Faits de langues, 28, 119-132.

Debaisieux, J.-M. Dir. (2013). Analyses linguistiques sur corpus - subordination et insubordination en français. Paris : Lavoisier.

Gettrup, H. \& H. Nolke. (1984). Stratégies concessives : une étude de six adverbes français. Revue romane, 19, 3-47.

Guimier, C. (2000). Non congruence et congruence : «alors que» vs. «tandis que ». Syntaxe et sémantique, 1, 79111.

Grevisse, M. \& A, Goosse. (2008). Le bon usage. 14ème édition, Gembloux : Duculot.

Lafontaine, F. (2012). Quelques apports sur le traitement syntaxique de la forme alors que. Tipa, 28, 18-38.

Lafontaine, F. (2013). Sur certains 'effets d'enchâssement' des constructions en alors que. Studia UBB Philologia, 58-4, 53-65.

Moeschler, J. \& Auchlin A. (2009). Introduction à la linguistique contemporaine. Paris : Armand Colin (Cursus), 3e édition.

Morel, M.-A. (1996). La concession en français. Paris : Ophrys.

Oversteegen, L.-E. (1997). On the pragmatic nature of causal and contrastive connectives. Discourse Processes, 24, $51-85$.

Piot, M. (1993). Les connecteurs du français de France. Linguisticae Investigationes, 17-1, 142-160.

Riegel, M., Pellat J.-C. et Rioul R. (2008). Grammaire méthodique du français. 5ème édition, Paris : PUF.

Roze, C. (2013). Vers une algèbre des relations de discours. Thèse de doctorat. Université Paris Diderot. 
Spooren, W. (1989). Some aspects of the form and interpretation of global contrastive coherence relations. Ph.D. thesis. K.U. Nijmegen.

Sabio, F. (2011). Syntaxe et organisation des énoncés - Observations sur la grammaire du français parlé. Mémoire d'habilitation à diriger des recherches, Université Aix-Marseille 1.

Sandfeld, K. (1965). Syntaxe du français contemporain. Les propositions subordonnées, Genève : Droz.

${ }^{1}$ « [...] les propositions circonstancielles sont introduites par des conjonctions (...) qui à la fois marquent leur caractère subordonné et spécifient leur rapport sémantique avec le reste de la phrase » (M. Riegel et al., 2008 : 475).

${ }^{2}$ Pour ce travail, nous avons interrogé plusieurs bases de données. Pour la partie écrite, le Corpus Evolutif de Référence du Français (CERF) fournit 9 millions de mots. En ce qui concerne l'oral, nous avons consulté le Corpus de Référence du Français Parlé (CRFP) réalisé par l'équipe DELIC ; le CRFP II, mis en œuvre par A. Valli ; et le Corpus de Langues parlées en Interaction (CLAPI) élaboré par le groupe ICOR.

${ }^{3}$ On se reportera à J.-M. Debaisieux (2013) à propos du caractère plurifonctionnel des conjonctions de subordination.

${ }^{4}$ On peut émettre une hypothèse concernant cette résistance à certains tests : ce blocage serait dû au sémantisme de la conjonction alors que. En effet, une particularité sémantique de alors que par rapport à d'autres conjonctions plus nettement temporelles telles que quand ou lorsque réside dans son caractère "non congruent», pour reprendre l'expression proposée par C. Guimier (2000:84).

${ }^{5}$ A la différence de la relation concessive, dans la relation contrastive, « les deux propriétés assignées aux différentes entités ou aux différents éléments reliés peuvent être incompatibles, mais elles ne résultent pas en une contradiction grâce au fait qu'elles ne sont pas assignées à la même entité, au même moment, et au même endroit. » Traduit de L. E. Oversteegen (1997) et cité par C. Roze (2013 : 71).

${ }^{6}$ Nous nous appuyons sur la définition de la concession établie par M.-A. Morel (1996: 7) : «lorsqu'on pose ensemble deux propositions $\mathrm{A}$ et $\mathrm{B}$ et que l'on considère que $\mathrm{B}$ se présente comme la négation du terme que l'on associe normalement à $\mathrm{A}$ (noté ici $\mathrm{B} 1$ ), on établit une relation concessive entre $\mathrm{A}$ et $\mathrm{B}$, quelles que soient la structure des énoncés $A$ et $B$ et la nature du lien formel qui les unit (bien que, même si, si... que, malgré, etc.). On posera donc que deux énoncés $\mathrm{A}$ et $\mathrm{B}$ sont en relation concessive si $\mathrm{A}$ est normalement associé à $\mathrm{B} 1 \mathrm{et} \mathrm{B}=$ non $\mathrm{B} 1$ ».

${ }^{7}$ Il s'agit là de cas non canoniques de réalisation d'une place syntaxique.

${ }^{8}$ K. Sandfeld, (1965 : 304) fait état de cette possibilité de changement modal pour la forme alors que et tandis que en relevant un exemple introduit par tandis que qui opère un passage de la modalité assertive à jussive : A ce jeu de voleurs, les chrétiens ne sont pas de force, ils finissent toujours par se noyer; tandis que prenez un juif... jetez-le dans l'eau trouble de quelque affaire véreuse, et il se sauvera (Z. Argent 93).

${ }^{9}$ Il s'agit de cas d'interrogations oratoires pour lesquelles «l'orientation argumentative de l'énoncé interrogatif est systématiquement identique à celle de l'énoncé négatif correspondant, et non à celle de l'assertion positive» (J. Moeschler, 2009 : 155) 